\title{
A LITERATURA SOBRE METODOLOGIAS ATIVAS EM EDUCAÇÃO MÉDICA NO BRASIL: NOTAS PARA UMA REFLEXÃO CRÍTICA
}

\author{
THE LITERATURE ON ACTIVE METHODOLOGIES IN MEDICAL \\ TRAINING IN BRAZIL: NOTES FOR A CRITICAL REFLECTION
}

Raimundo Rodrigues de França Junior ${ }_{\text {(https://orcid.org/0000-0002-3530-8940)', }}^{1}$
Marlécio Maknamara ${ }_{\text {(https://orcid.org/0000-0003-0424-5657)² }}^{2}$

${ }^{1}$ Universidade Federal de Alagoas, Faculdade de Medicina, Arapiraca, Alagoas, Brasil. $<$ raimundoufal@yahoo.com.br>

${ }^{2}$ Universidade Federal da Bahia, Programa de Pós-Graduação em Educação, Salvador, Bahia, Brasil.

Resumo Este trabalho parte da emergência de escolas médicas que fundamentam seus currículos nas metodologias ativas. Teve como objetivo realizar um mapeamento na Revista Brasileira de Educação Médica com base na produção científica sobre conexões entre formação médica e metodologias ativas. O levantamento bibliográfico incluiu 53 artigos, agrupados em quatro categorias de análise: análise comparativa entre modelos curriculares fundamentados no método tradicional e nas metodologias ativas; avaliação da mudança curricular baseada no uso das metodologias ativas; avaliação do desempenho discente com uso das metodologias ativas; e capacitação docente no contexto das metodologias ativas. Esses resultados apontam as metodologias ativas como propostas que podem dar respostas aos anseios de mudança curricular dos cursos de medicina no Brasil, ainda que não exista um consenso em relação à efetividade do seu uso nessa área. Todavia, um olhar mais detalhado sobre esses resultados nos permitiu concluir que esse processo, quando baseado apenas na mudança do método de ensino, pode apresenta lacunas e mostra não ser suficiente para mudança do perfil do egresso em medicina. Desse modo, recomendamos serem realizadas mais pesquisas nesse campo para melhor compreender os usos das metodologias ativas nas dinâmicas curriculares que fundamentam a educação médica no Brasil. Palavras-chave educação médica; aprendizagem baseada em problemas; problematização.

\begin{abstract}
The study begins with the emergence of medical schools that base their syllabuses on active methodologies. It had the goal of mapping Revista Brasileira de Educação Médica (Brazilian Journal of Medical Training) based on the scientific articles about the connections between medical training and active methodologies. The bibliographic survey included 53 articles, which were grouped into four categories of analysis: comparative analysis of the syllabus models based on the traditional method and on the active methodologies; assessment of the syllabus change based on the use of active methodologies; assessment of professor performance with the use of active methodologies; and student training in the context of the active methodologies. The results indicate that the active methodologies are proposals that may fulfil the wish to promote syllabus changes in the medicine courses in Brazil, even though there still is not a consensus regarding the effectiveness of its use in this field. However, a closer look at the results enabled us to conclude that this process, when based only on the change in teaching method, may present gaps and proves insufficient to change the profile of the medicine graduates. Thus, we recommend the conduction of further researches in this field in order to better understand the uses of the active methodologies in the syllabus dynamics that form the basis of medical training in Brazil.
\end{abstract}

Keywords medical training; learning based on problems; problematization.

(cc) BY Este é um artigo publicado em acesso aberto sob uma licença Creative Commons. 


\section{Introdução}

Nos últimos sessenta anos, a educação médica encontra-se sob crítica recorrente em todo o mundo. No Brasil, especialmente nas últimas duas décadas, tornou-se objeto privilegiado de análise e debates pelos profissionais da área e pela sociedade em geral. Segundo Nogueira (2009, p. 02) “existe, de certa forma, um consenso quanto à necessidade de reformulação de determinados aspectos da formação médica com vistas a uma satisfação das demandas assistenciais em saúde atuais".

Frente a essa perspectiva, muitos programas (a exemplo do Programa de Incentivo às Mudanças nos Cursos de Medicina - Promed ${ }^{1}$ ) e pesquisas como, por exemplo, Araújo e Sastre (2009), Decker e Bouhuijs (2009); Vila e Vila (2007); Carabetta Júnior (2016); Farias (2015), Mitre et al. (2008), Nunes e Nunes (2005), Nogueira (2009), Penaforte(2001) e Silva et al. (2015) - têm apontado como aspecto para o alcance dessa demanda, uma formação que incorpore metodologias ativas (MA) no processo ensino e aprendizagem de estudantes da saúde, e em específico, da medicina. Essas pesquisas têm reiterado o discurso das MAs como prática ideal para formação de um profissional mais adequado ao ato médico e às práticas de saúde, tal como requerido pelas Diretrizes Curriculares Nacionais (DCNs) de medicina (Brasil, 2014).

Esses debates e discursos que envolvem as MAs - apontados muitas vezes pelos autores aqui citados como uma possibilidade e até mesmo como uma emergência na formação médica -, somados ao aumento do número de escolas médicas que fundamentam seus currículos nas MAs, nos últimos vinte anos no Brasil, nos inspiram a realizar o presente levantamento bibliográfico, conectando educação médica ${ }^{2}$ e as MAs. O objetivo foi produzir um panorama de como se encontram as pesquisas sobre o uso das MAs na formação médica no Brasil, privilegiando a produção da Associação Nacional de Pós-Graduação e Pesquisa em Educação (Anped) e da Revista Brasileira de Educação Médica (RBEM) para tanto.

\section{Uma possibilidade para a formação médica}

Nas últimas duas décadas, as discussões no campo da educação médica no Brasil têm enfatizado questões como proposta curricular e modelo pedagógico. Nunes e Nunes (2005) afirmam que isso ocorre em virtude de a educação hoje exigir postura aberta a mudanças e revisão de paradigmas. A adoção desse tipo de postura é justificada pelos pesquisadores da área, pela necessidade de superar um modelo tradicional de ensino, fundamentado principalmente no flexnerianismo. Vale lembrar que esse modelo (orientador da reforma universitária de 1968) foi responsável por focar o aprendizado na 
transmissão do conhecimento pelo professor e tornar obrigatório o ensino centrado no hospital, oficializar a separação entre ciclo básico e profissional, e imprimir características mecanicistas, biologicistas e individualizantes ao ensino médico (Nogueira, 2009).

Objetivando superar essa fragmentação do conhecimento científico, ganha cada vez mais destaque o discurso sobre a necessidade de que a organização curricular e o conteúdo programático desenvolvido nas escolas médicas assumam uma proposta interdisciplinar. Tal proposta visa desvincular-se "de um currículo fechado e estanque, conteudista, biologicista, com pouca, ou nenhuma, relação entre as diferentes áreas do conhecimento e ausente de uma visão unificada do corpo humano" (Carabetta Júnior, 2016, p. 114). Esse é um tipo de discurso que se justifica pela afirmativa de que hoje “o acúmulo de informações de nada vale se estiver isolado da capacidade de resolver novos problemas que surgem diariamente" (Nunes e Nunes, 2005, p. 180).

A esse discurso é acrescentada a orientação de que os objetivos educacionais desses currículos sejam definidos de acordo com a perspectiva do construtivismo,

[...] em que a aprendizagem passa a ser caracterizada como uma construção realizada pelo sujeito por meio das relações que estabelece entre as informações que lhe são apresentadas, entre elas com seus conhecimentos prévios e com seu meio social, o que implica uma nova realidade educacional com a ação do aluno para a criação de algo novo por meio do próprio fazer. (Carabetta Júnior, 2016, p. 114).

Nesse contexto, as exigências são de um trabalho em que a aprendizagem seja 'ativa', isto é, "construída pelo aluno a partir de interações dialógicas com o professor, com os colegas e com os diferentes conteúdos" (Carabetta Júnior, 2016, p. 114). Observamos com isso o deslocamento do foco da 'simples reprodução de conhecimentos' para o desenvolvimento de competências e habilidades "que devem caminhar junto com a construção do conhecimento socialmente estabelecido" (Carabetta Júnior, 2016, p. 115). Ao buscar atender a essas recomendações, coloca-se em pauta nas discussões sobre educação médica no Brasil a necessidade de reformulação curricular e do modelo pedagógico.

Esse processo de reformulação é orientado pela resolução n. 03 de 20/06/2014 do Ministério da Educação que institui as DCNs para a graduação em medicina. Destacamos aqui o capítulo III dessa resolução que institui recomendações sobre os Conteúdos Curriculares e o Projeto Pedagógico do Curso de Graduação em Medicina. No artigo 26, a resolução estabelece que o curso deverá ser "centrado no aluno como sujeito da aprendizagem e apoiado no professor como facilitador e mediador do processo, com vistas à formação integral e adequada do estudante" (Brasil, 2014, p. 12). Ao buscar garantir essa postura na relação professor-aluno, no art. 29, item II e IV, orienta-se 
que o curso deve "utilizar metodologias que privilegiem a participação ativa do aluno na construção do conhecimento e na integração entre os conteúdos, assegurando a indissociabilidade do ensino, pesquisa e extensão", bem como "promover a integração e a interdisciplinaridade em coerência com o eixo de desenvolvimento curricular" (Brasil, 2014, p. 12).

Essas DCN nos revelam que as exigências a uma proposta curricular e a um modelo pedagógico devem ir além da simples ilustração (Nogueira, 2009), requerendo dos atores envolvidos no processo educacional a capacidade criativo-pragmática. Em decorrência dessa constatação, tem-se buscado cada vez mais colocar em prática metodologias de ensino e aprendizagem com possibilidade de desenvolver nos alunos tais competências (Nunes e Nunes, 2005). Essa demanda tem levado muitas pesquisas no campo da educação médica a afirmarem que as MAs estão mais adequadas ao perfil de profissional que se almeja.

Os discursos em torno dessas MAs indicam que elas admitem uma prática pedagógica "ética, crítica, reflexiva e transformadora, ultrapassando os limites do treinamento puramente técnico, para efetivamente alcançar a formação do homem como um ser histórico, inscrito na dialética da ação-reflexão-ação" (Mitre et al., 2008, p. 10). Nessa mesma linha de raciocínio abordada por Mitre et al. (2008), Farias (2015) aponta as MAs como um processo em que os estudantes desenvolvem atividades as quais necessitam de reflexão de ideias e desenvolvimento da capacidade de usá-las. Ademais, define que para ser considerado bom método, ele deve ser:

\footnotetext{
Construtivista - se basear em aprendizagem significativa; Colaborativo - favorecer a construção do conhecimento em grupo; Interdisciplinar - proporcionar atividades integradas a outras disciplinas; Contextualizado - permitir que o educando entenda a aplicação deste conhecimento na realidade; Reflexivo - fortalecer os princípios da ética e de valores morais; Crítico - estimular o educando a buscar aprofundamento de modo a entender as limitações das informações que chegam até ele; Investigativo - despertar a curiosidade e a autonomia, possibilitando ao educando a oportunidade de aprender a aprender; Humanista - ser preocupado e integrado ao contexto social; Motivador - trabalhar e valorizar a emoção; Desafiador - estimular o estudante a buscar soluções (Farias, 2015, p. 146).
}

Farias (2015) afirma existir uma infinidade de métodos ativos de educação, sendo comum, na educação médica, referências a Aprendizagem Baseada em Problemas (ABP - do inglês Problem Based Learning - PBL) e a Problematização. De acordo com Nunes e Nunes (2005), essas MAs guardam estreita relação com os pressupostos filosófico-pedagógicos de John Dewey e Paulo Freire, respectivamente, pois visam ao desenvolvimento de cidadãos cons- 
cientes das realidades vividas e participantes de transformações baseadas num senso crítico.

Dewey (1959), ao afirmar a necessidade de romper com a postura de mera transmissão de informações, na qual os estudantes assumem o papel de receptáculos passivos, preocupados apenas em memorizar conteúdos e recuperá-los quando solicitado — habitualmente, por ocasião de uma prova - , orienta-nos sobre a possibilidade da ascensão da $\mathrm{ABP}$ na educação médica. De fato, um dos aspectos cruciais da ABP é o processo educativo centrado no estudante, que permite que este seja capaz de se 'tornar maduro', e adquira graus crescentes de autonomia.

É com base nessa perspectiva que os objetivos educacionais na ABP foram definidos, com ênfase no "desenvolvimento das habilidades do pensamento crítico, do entendimento, do aprender a aprender e do trabalho grupal e cooperativo" (Decker e Bouhuijs, 2009, p. 187). Segundo Putnam (2001), os objetivos educacionais da ABP direcionam os alunos para:

desenvolver uma abordagem sistemática para a solução de problemas da vida real utilizando habilidades mentais superiores, como aquelas relacionadas à resolução de problemas, ao pensamento crítico e à tomada de decisões; adquirir uma base ampla de conhecimentos integrados, que possam ser acessados e aplicados a diferentes situações; desenvolver habilidades para a aprendizagem autodirigida, identificando o que é preciso aprender, localizando e utilizando recursos apropriados, aplicando a nova informação/conhecimento na resolução de problemas [...]; desenvolver atitudes e habilidades necessárias para o trabalho efetivo em equipe [...]; adquirir o hábito permanente de abordar um problema com iniciativa e diligência, mantendo a propensão para aquisição dos novos conhecimentos e habilidades necessários para a sua resolução; desenvolver o hábito de autorreflexão e autoavaliação, que lhes permita considerar honestamente seus pontos fortes e suas fraquezas, bem como o estabelecimento de metas realísticas. (Putnam, 2001, p. 7, tradução nossa).

Seguindo esse mesmo entendimento, Araújo e Sastre (2009) afirmam que na $\mathrm{ABP}$ o aluno parte de problemas ou situações que objetivam gerar dúvidas, desequilíbrios ou perturbações intelectuais, com forte motivação prática e estímulo cognitivo para evocar as reflexões necessárias à busca de adequadas escolhas e soluções criativas. Isso pode, segundo Penaforte(2001), estabelecer uma aproximação à proposta educativa formulada por John Dewey. Ademais, a ABP se inscreve, de acordo com Penaforte(2001), em uma perspectiva construtivista - relacionada, especialmente, aos referenciais da teoria piagetiana da equilibração e desequilibração cognitiva (Penaforte, 2001). Tal perspectiva considera que o conhecimento deve ser produzido na interseção entre sujeito 
e mundo, como amplamente problematizado por teóricos como Paulo Freire (1987; 1999).

A perspectiva freiriana sobre uma "educação libertadora" (Freire, 1987, p. 34) serviu como fundamento, em específico, para a metodologia da problematização, a qual tem como meta "a mobilização do potencial social, político e ético dos alunos, que estudam cientificamente para agir politicamente, como cidadãos e profissionais em formação, como agentes sociais que participam da construção da história de seu tempo, mesmo que em pequena dimensão" (Berbel, 1998, p. 7). Essa meta vai ao encontro das proposições de uma pedagogia crítica, que "define educação como atividade em que professores e alunos são mediatizados pela realidade que apreendem e da qual extraem o conteúdo da aprendizagem, atingem um nível de consciência dessa realidade, possibilitando a transformação social" (Vila e Vila, 2007, p. 180).

Com base nessa assimilação, observamos que os conceitos de conscientização, emancipação, transformação social, somados aos de autoconhecimento e autorreflexão (Decker e Bouhuijs, 2009), são tidos como essenciais na definição de objetivos educacionais para dirigir o processo de ensino-aprendizagem na metodologia da problematização. Sobre esse processo, Nunes e Nunes (2005), ao trabalhar com o pensamento de Paulo Freire, propõem alguns passos para o aprendizado:

A partir da vivência real dos educandos, são identificados pelo educador os temas geradores (assuntos de grande interesse dos educandos em seu cotidiano) que, nesse primeiro momento, se encontram ainda não problematizados. Esses temas geradores são apresentados para os educandos que realizam um trabalho de problematização desses temas geradores, isto é, realizam uma contextualização desses temas em suas vivências. A partir dessa problematização, busca-se fundamentação teórica na tentativa de entender os problemas levantados. Os alunos realizam, então, uma reflexão crítica, num processo dialógico entre educadores-educandos, realizado também em pequenos grupos, tentando identificar quais as causas histórico-sociais desse problema, e a partir dessa tomada de consciência propõem uma ação social para alcançar uma mudança da realidade (p. 181).

Esses passos estão intrinsecamente relacionados ao 'método do arco de Charles Maguerez'3 (Bordenave e Pereira, 2007), que, segundo Berbel(1998), é a primeira referência para a metodologia da problematização. Em síntese, a autora afirma que essa metodologia tem uma orientação geral como todo método, com etapas distintas e encadeadas mediante um problema detectado na realidade, e estaria voltada para "preparar o estudante/ser humano para tomar consciência de seu mundo e atuar intencionalmente para transformálo" (p. 144). 
Essas discussões de cunho teórico sob as quais estão assentadas as MAs, em específico a ABP e a Problematização, apontam o uso dessas metodologias como um potencial para formar um profissional 'crítico', 'reflexivo', 'humanista', 'ético' e 'transformador', tal como demandado pelas DCNs de medicina (Brasil, 2014). O desejo de observar como essa proposta vem se desenvolvendo nas escolas de medicina, nos levou a realizar o presente levantamento bibliográfico sobre o uso dessas MAs na educação médica. O primeiro passo para realização dessa tarefa foi demarcar um caminho metodológico que pudesse nos orientar na busca de informações, o qual será descrito no tópico a seguir.

\section{Buscar, selecionar, inferir e interpretar: apontando procedimentos da pesquisa}

O caminho metodológico percorrido para realização desse trabalho ${ }^{4}$ entendido aqui como um mapeamento sobre a produção científica de um tema específico - inspira-se nos critérios de uma pesquisa bibliográfica. De acordo com Gil (2002), esse tipo de pesquisa é desenvolvido com base em material já elaborado, constituído principalmente de livros e artigos científicos. A principal vantagem desse tipo de pesquisa, segundo esse autor, reside no fato de "permitir ao investigador a cobertura de uma gama de fenômenos muito mais ampla do que aquela que poderia pesquisar diretamente" (Gil, 2002, p. 45).

Foram buscadas as produções textuais (trabalhos e pôsteres) nos GTs 04 (Didática) e 12 (Currículo) da $24^{\mathrm{a}}$ à $38^{\mathrm{a}}$ reunião anual da Associação Nacional de Pós-Graduação e Pesquisa em Educação (Anped), além das publicações dos volumes da Revista Brasileira de Educação Médica (RBEM) no período de 2001 a $2017^{5}$.

A busca e a seleção das produções nas referidas bases de dados foram inspiradas no trabalho de Bardin (1977): a pré-análise; a exploração do material coletado; e o tratamento das informações obtidas, a inferência e a interpretação. De acordo com esse autor, a pré-análise é o momento inicial da pesquisa, caracterizada pelo uso de palavras-chave para seleção de trabalhos (a serem submetidos à análise) que tenham como foco o tema pesquisado. Nesse trabalho, a identificação das produções foi feita com base nas seguintes palavras-chave presentes no título, resumo e corpo do texto: educação médica; metodologias ativas; Aprendizagem Baseada em Problemas (ABP) ou Problem Based Learning ( $P B L$ ) e problematização.

Após esse procedimento, não foi encontrado nenhum trabalho nas reuniões da Anped. Na RBEM, dos 994 artigos do período avaliado, 87 foram selecionados pelas palavras-chave, distribuídos entre todas as seções existentes na revista. Após essa seleção, foi realizada uma 'leitura flutuante' (Bardin, 1977) do texto completo, o que resultou na exclusão de 34 produções (apesar de 
terem as palavras-chave buscadas, não abordavam as MAs na educação médica de forma direta). Ao final, o levantamento foi constituído por um corpus de 53 trabalhos.

Na segunda etapa, esses trabalhos selecionados foram lidos na íntegra, fichados e submetidos à categorização, isto é, "processo pelo qual os dados brutos são transformados e agregados em unidades que permitem uma descrição exata das características relevantes do conteúdo" (Bardin, 1977, p. 103). Depois, foi realizado o tratamento dos resultados obtidos com base em inferências e interpretações, o que para Bardin (1977) constitui a terceira etapa de um estudo bibliográfico. Vale salientar que, para efeito de conceituação, a condução de todas as etapas mencionadas foi inspirada em elementos da 'análise de conteúdo' com ênfase na modalidade de 'análise temática', que segundo Gerhardt et al. (2009), de um ponto de vista operacional, inicia-se pela 'leitura das falas'.

Nesse procedimento, as falas presentes nas produções foram lidas, com destaque para os resultados que correspondem a afirmações sobre o a conexão entre educação médica e o uso das MAs. A análise temática permite "descobrir os núcleos de sentido que compõem uma comunicação cuja presença ou frequência signifique alguma coisa para o objetivo analítico visado" (Minayo, 2007, p. 316).

\section{O que diz a literatura sobre o uso das MAs em educação médica no Brasil}

Depois da leitura na íntegra dos trabalhos selecionados na base de dados da RBEM, foi possível agrupá-los em quatro subtemas ou categorias: análise comparativa (comparação entre modelos curriculares fundamentados no método tradicional e nas MAs); avaliação da mudança curricular (uso das MAs); avaliação do ensino e aprendizagem (desempenho discente com uso das MAs); e capacitação docente (capacitação docente no contexto das MAs).

\section{Análise comparativa entre modelos curriculares}

Do total de produções selecionadas, sete (13\%) abordaram uma análise comparativa entre os modelos curriculares tradicional e o fundamentado nas MAs (Araújo et al., 2001; Lima Júnior, 2002; Lima, 2005; Souza e Menezes, 2005; Gomes et al., 2009; Peixoto, 2011; e Tenório et al., 2016).

Lima Júnior (2002) e Gomes et al. (2009) analisaram estudos que comparam o emprego do modelo educacional tradicional e do modelo fundamentado nas MAs na formação médica. Seus resultados indicaram, com base em uma reflexão de cunho teórico sobre a mudança de paradigma na educação 
médica, que o modelo tradicional não tem se mostrado eficaz para os novos desafios da educação médica. De modo contrário, o modelo fundamentado nas MAs é considerado eficaz e potente para atender a esses desafios, e dentre os métodos de ensino, a ABP ganha destaque. A análise de Gomes et al.(2009) sobre estudos que comparam a o uso da ABP na formação médica com o currículo tradicional concluiu que a $\mathrm{ABP}$ é uma alternativa na implementação das DCNs de medicina.

Estudos empíricos (Araújo et al., 2001; Lima, 2005; Peixoto, 2011) também avaliaram diferenças no rendimento cognitivo sobre conteúdo específico e desempenho de habilidades e atitudes de alunos, segundo os modelos de currículos (o tradicional e o fundamentado nas MAs).

Na investigação de Araújo et al. (2001) foram identificadas diferenças no aproveitamento cognitivo do conteúdo curricular de nefrologia, em dois grupos de alunos da Faculdade de Medicina de Marília (Famema), inseridos em modelos curriculares diferentes. Baseado em testes de múltipla escolha, houve um aproveitamento médio inferior dos alunos inseridos em um currículo orientado pela $\mathrm{ABP}$, quando comparados aos alunos inseridos em um modelo curricular tradicional.

Lima (2005) objetivou verificar se existem diferenças na habilidade de buscas de informações entre médicos graduados pela metodologia tradicional e ABP da Famema. Nesse estudo, um questionário com perguntas fechadas sobre as experiências em uso de fontes de informação identificou que os graduandos em medicina pela metodologia ABP relatam maior facilidade no uso dos recursos informacionais em comparação àqueles que se graduaram pela metodologia tradicional. Peixoto (2011) comparou a atitude do estudante de medicina a respeito da relação médico-paciente de uma escola com currículo abalizado em métodos tradicionais com outra em ABP. A coleta de dados foi realizada com a aplicação da escala Patient-Practitioner Orientation Scale (PPOS). Atitudes mais centradas no paciente foram encontradas em estudantes da escola com currículo fundamentado na ABP; já a deficiência de sensibilidade e empatia se mostrou mais presente em estudantes da escola com currículo baseado nos métodos tradicionais de ensino.

Os trabalhos mencionados mostram que o desempenho de determinadas habilidades e atitudes médicas se tornam mais presentes em alunos inseridos em um currículo orientado pelas Mas. A isso é somada a importância que os estudos teóricos atribuem às MAs, em específico à $\mathrm{ABP}$, para a formação médica. Todavia, estudo de Araújo et al., 2001 demonstrou um menor desempenho cognitivo em alunos inseridos em um currículo orientado pelas MAs. Parece ainda não haver um consenso em relação à efetividade no uso das MAs para a formação médica. 


\section{Avaliação da mudança curricular fundamentada no uso das MAs}

Dos 53 trabalhos selecionados, 19 pesquisas (36\%) avaliam o processo de implementação das MAs nos currículos médicos, nas visões docente e discente desse processo (Ferreira Filho et al., 2002; Turini e Almeida, 2002; Wierzchon, 2002; Moré e Gordan, 2004; Barros e Lourenço, 2006; Moraes e Manzini, 2006; Chaves e Grosseman, 2007; Gomes et al. 2008; Vargas et al., 2008; Aguilar-da-Silva, 2009; Cezar, 2010; Costa et al., 2011; Mezzaril, 2011; Brandão et al., 2013; Gomes e Rego, 2014; Maia, 2014; Luna e Bernardes, 2015; Cliquet e Rodrigues, 2016; Sena e Costa, 2016).

Autores como Wierzchon (2002), Aguilar-da-Silva (2009) e Gomes e Rego (2014) discutem, desde uma perspectiva teórico-bibliográfica de investigação, o processo de mudança curricular em medicina, com foco na implementação das MAs, e em específico a ABP. Entre esses autores, é unânime a ideia de que a $\mathrm{ABP}$ é uma boa alternativa para formar um profissional capaz de tratar o indivíduo doente como um todo, observando todos os aspectos relevantes para a saúde.

Todavia, é unânime também a ideia de que somente a mudança do método tradicional para o $\mathrm{ABP}$ não é suficiente para garantir a transformação na educação médica e fomentar isoladamente a mudança no perfil do egresso. Esses trabalhos mostram que a concepção bancária de educação não está vinculada necessariamente à transmissão de conteúdos em uma sala de aula expositiva, como é recorrente no método tradicional de ensino. Ela pode se dar de forma efetiva "a partir também do interesse da seleção de conteúdos de uma situação-problema e do docente que orienta o seu processamento a partir da ABP" (Gomes e Rego, 2014, p. 04).

Os estudos empíricos que buscaram avaliar esse processo de mudança curricular em escolas médicas com MAs, apontam para os mesmos fatos observados desde a teoria. A insuficiência da ABP como método para garantir a mudança no perfil do egresso foi abordada nesses estudos, os quais indicam a necessidade de associar outros métodos à $\mathrm{ABP}$, como a Problematização.

Brandão et al. (2013) objetivaram descrever um relato de experiência das vivências das Práticas de Integração Ensino-Serviço-Comunidade (Piesc) como eixo de apoio do modelo de reorientação na formação médica da Universidade Estadual do Sudoeste da Bahia. O resultado de sua investigação indicou que as Piesc, como eixo de apoio da $\mathrm{ABP}$, conseguem articular os saberes com as práticas na reorientação da formação médica.

O trabalho de Moraes e Manzini (2006) analisou as concepções de docentes, gestores e estudantes do curso de medicina da Famema sobre a ABP e sua relação com a formação médica. Para coleta de material empírico com os sujeitos investigados, foram realizadas entrevistas semiestruturadas, submetidas, posteriormente, à análise de conteúdo temática. Os resultados da pesquisa 
apontaram para o fato de que mudar um currículo e o método de ensino e aprendizagem não garante a mudança de concepções e práticas dos professores e estudantes. A esse processo de mudança deve ser somado investimento em educação permanente para os docentes, que precisa ser estendido à prática profissional dos trabalhadores da saúde.

Nos trabalhos mencionados, a ABP é percebida como uma possibilidade para iniciar o processo de mudança curricular do tradicional para MAs. Entretanto, esse método não garante por si só a mudança no perfil do egresso em medicina. Os resultados das investigações aqui apresentadas indicam, em parte, ser necessário somar outros fatores à adoção da ABP, como investimento em educação permanente, e associação de outros métodos à $\mathrm{ABP}$, como a Problematização.

\section{Avaliação do ensino e aprendizagem baseado nas MAs}

Chamou atenção o número expressivo de trabalhos que buscaram discutir o desempenho discente com uso das MAs na educação médica. Dos 53 trabalhos encontrados, 18 (34\%) abordaram esse tema (Angeli e Loureiro, 2001; Goulart et al., 2001; Lima e Linhares, 2008; Komatsu, 2009; Gomes, 2010; Marin, et al., 2010; Novaes et al. 2010; Andrade et al. 201 1; Aquilante et al., 2011; Gomes e Rego, 2011; Oliveira e Batista, 2012; Costa et al, 2014; Makabe e Maia, 2014; Smolka et al., 2014; Ferreira et al., 2015; Leon e Onófre, 2015; Costa, et al. 2016; Taroco, 2017).

Gomes e Rego (2011), em uma pesquisa teórico-bibliográfica, avaliaram os resultados do uso da $\mathrm{ABP}$ na formação médica nos últimos vinte anos de forma a compor um panorama da sua efetividade na busca do médico crítico, reflexivo e com responsabilidade social. Os resultados da pesquisa apontaram para a insuficiência da mudança pedagógica isolada como resposta a uma formação médica capaz de aliar competências técnicas e ético-humanísticas. Esses autores nos chamam atenção ainda para a escassez de dados fundamentados sobre o padrão de desempenho dos graduandos em medicina no Brasil, e afirmam que isso dificulta a avaliação e análise objetiva da eficácia e efetividade de currículos fundamentados na ABP.

Marin et al. (2010) apontam resultados empíricos que parecem indicar possíveis razões para a conclusão de Gomes e Rego (2011) em seu estudo da literatura. Marin et al. (2010) entrevistaram discentes sobre as fortalezas e fragilidades dos métodos ativos de aprendizagem. A análise desse material foi guiada pela 'hermenêutica dialética', tendo como referência os princípios do 'método de interpretação de sentidos'. Seus resultados apontaram para o fato de que as MAs estimulam o estudo constante, a independência e a responsabilidade, possibilitam a integração das dimensões biopsicossociais, 
preparam para o trabalho em equipe e aproximam os alunos dos usuários e da equipe. No entanto, esses autores afirmam que os sentidos extraídos das falas dos estudantes indicam que nem todos estão preparados para isso; algumas vezes, sentem-se perdidos em busca de conhecimentos, além de apresentarem dificuldades quanto a sua inserção na equipe de saúde.

Tais apontamentos corroboram com o trabalho de Angeli e Loureiro(2001), que buscaram investigar como estudantes do curso médico da Famema avaliam sua experiência de aprendizagem por meio da ABP. Para coleta de informações, foram realizados grupos de reflexão conforme metodologia proposta por Delarossa (1979). Os resultados da pesquisa indicam que o êxito da implantação dessa metodologia pode depender, em parte, dos recursos pessoais de adaptação dos estudantes (características de personalidade como independência, determinação, senso de responsabilidade, desinibição, capacidade de comunicação e de organização).

Andrade et al. (2011) analisaram a aquisição e evolução de atitudes humanísticas dos discentes do curso de medicina da Escola Superior de Ciências da Saúde - que tem seu currículo fundamentado na ABP. Foram utilizados dois instrumentos para coleta: a Escala de Atitude de Estudantes de Medicina (do tipo Likert) e um questionário sociodemográfico semiestruturado e autoaplicável (confeccionado pelos pesquisadores). Embora as atitudes positivas tenham predominado em relação ao tema investigado, o autor ressalta a necessidade de uma formação continuada do docente como condição para um bom desempenho discente e para o êxito na implementação de um currículo fundamentado nas MAs.

De modo geral, os resultados de investigação dos trabalhos já mencionados mostram existir dificuldades em se avaliar o desempenho discente quando inseridos em uma proposta curricular baseada nas MAs, haja vista a escassez de dados fundamentados. Contudo, na tentativa de realizar avaliações dessa natureza, esses mesmos trabalhos apontaram a insuficiência do método ABP para a mudança do perfil do egresso de medicina, tal como discutido no tópico anterior. Em um currículo orientado pelas MAs, o desempenho discente está condicionado, em parte, pelos 'recursos pessoais de adaptação' assim como pela formação continuada do docente.

\section{Capacitação docente no contexto das MAs}

O entendimento da capacitação docente como condição para o êxito na implementação das MAs em um currículo médico foi apontado em quase todas as produções bibliográficas, e mais diretamente em nove (17\%) dos 53 trabalhos selecionados (Venturelli e Fiorini, 2001; Almeida et al., 2003; Lima 
et al., 2003; Almeida e Ferreira Filho, 2008; Arruda et al., 2008; Faria et al. 2008; Almeida e Batista, 2011; Rego e Batista, 2012; Almeida e Batista, 2013).

Considerando especificamente a área médica, alguns autores assumem a capacitação docente como um componente essencial nos processos de mudanças curriculares e de sua consolidação. Lima et al. (2003) investigaram ações desenvolvidas pela Comissão de Capacitação Docente (CCD) do curso médico da Universidade Estadual de Londrina (UEL) com base em consultas ao arquivo dos registros de ações da CCD e análise das atividades desenvolvidas. Os resultados dessa análise apontaram como positivo o trabalho desenvolvido pela comissão e conclui ser pertinente um programa de capacitação mais abrangente, que envolva as instâncias gerenciais, profissionais e pedagógicas do processo.

Almeida et al. (2003) reiteram essa afirmativa, ao analisarem as perspectivas dos docentes da UEL que já atuaram como tutores acerca da capacitação recebida, mediante aplicação de um questionário estruturado fechado, com opções em escala tipo Likert. Os dados permitiram concluir que é necessário ampliar a capacitação em um processo de educação permanente que garanta a plena implantação e a sustentabilidade do novo currículo.

Essa mesma perspectiva é reafirmada por Almeida e Ferreira Filho(2008), quando analisam a experiência de docentes do curso de medicina da UEL que desenvolveram atividades não sistematizadas de educação permanente nos primeiros anos de implantação do currículo integrado. Os resultados da análise indicaram que as atividades desenvolvidas responderam demandas locais e contribuíram com a dinâmica de um processo de implantação que deve permanecer em contínua renovação.

Nessa mesma linha de raciocínio, Venturelli e Fiorini(2001) desenvolveram um estudo teórico sobre capacitação docente e educação médica e concluíram que a capacitação dos agentes comprometidos com os novos modelos educacionais (sejam eles docentes ou gestores) deve reconhecer que:

[...] o alto nível de qualificação que se espera dos docentes e os múltiplos papéis que eles podem desempenhar implicam o reconhecimento de que já não é suficiente apenas ter experiência numa área do conhecimento médico/ tecnológico tradicional para ser docente. 'Docência não é transferência de informação', indicou Paulo Freire (Pedagogia da Autonomia). É preciso ter uma sólida aprendizagem educacional, que, ademais, precisa ser mantida e atualizada (Venturelli e Fiorini, 2001, p. 11).

Ainda sobre esse tema, Almeida e Batista(2011) realizam um mapeamento das dificuldades e necessidades relativas ao desenvolvimento docente de professores atuantes em currículos que empregam as MAs na formação médica. A pesquisa foi realizada com os docentes da Universidade Estadual de 
Montes Claros/Unimontes, que trabalha com a ABP e a Problematização, por meio de questionários com perguntas fechadas e entrevistas semiestruturadas, submetidos à análise temática. Os resultados dessa investigação apontaram, como maior dificuldade, a insuficiência de investimento econômico por parte das instituições de ensino nesse processo de capacitação.

Por fim, como afirmado pelos resultados de investigação dos trabalhos analisados, considera-se plenamente estabelecido que os processos de inovações curriculares na educação médica exigem programas de capacitação docente permanentes e eficientes. Contudo, as instituições de ensino parecem não ter despertado para esse fato, uma vez que o investimento na formação docente é apontado por esses trabalhos como insuficiente, o que pode resultar em um menor avanço nas transformações.

\section{Considerações Finais}

O objetivo deste trabalho foi mapear a conexão entre educação médica e as MAs por meio de levantamento da produção bibliográfica no período de 2001 a 2017. Observamos que esses temas não aparecem em nenhuma produção da Anped no período destacado, o que constitui uma recusa dessa discussão, por parte da comunidade de pesquisadores em educação, no referido espaço.

No nosso entender, isso representa uma perda para ambas as partes, uma vez que as discussões sobre o uso das MAs na educação médica não são compartilhadas na Anped, e o debate dessa área de estudo fica ausente do maior evento científico da área educacional da América Latina. Esse fato configura-se, portanto, como um prejuízo, em termos de qualidade, sobre o que os pesquisadores da área da educação médica vêm conseguindo discutir sobre o uso das MAs.

Essa ausência de diálogo pode ser justificada por haver uma importante fração dos educadores brasileiros que apresentam fortes críticas às mudanças curriculares e a própria adesão às MAs na educação médica (Gomes e Rego, 2011). As produções sobre o tema estariam vinculadas, desse modo, a bases de dados como a RBEM, um espaço para a publicação de reflexões sobre um movimento de reformas curriculares ao qual a Associação Brasileira de Educação Médica (Abem) adere. Isso nos leva a crer que, o que está em jogo com essa ausência de diálogo são disputas entre diferentes concepções sobre as MAs, e relações de poder que privilegiam espaços de divulgação de saberes que reiteram uma verdade sobre o uso das MAs na educação médica.

Desse modo, podemos afirmar que essa recusa de um diálogo é interessada, e seus efeitos seriam a produção de discursos que passam a ser autorizados para falar de ensino e aprendizagem no âmbito da educação médica. As próprias categorias definidas com base na leitura dos artigos publicados pela RBEM 
demonstram como se dá essa produção discursiva, a qual reitera a necessária adoção das MAs. Isso explica que haja tantas produções que versam sobre sua adequada aplicação, avaliação do impacto na aprendizagem e adequação dos docentes para colocá-las em prática.

É válido apontar que essas produções que trataram da conexão entre os temas educação médica e as MAs correspondem a 5\% $(\mathrm{n}=53)$ do total de 994 trabalhos publicados pela RBEM no período investigado. Ao fazer uma leitura desses artigos, o que primeiro nos chamou atenção foi que a maioria usa os conceitos de educação e currículo. Observou-se o não cuidado, em termos de referenciamento adequado, quanto ao uso desses conceitos, na medida em que são citados, mas não discutidos.

Citar o conceito educação e associá-lo a um processo de mudança requer referenciá-lo e muitos teóricos contemporâneos fazem essa discussão, como Freire (1987; 1999), que discute educação como um processo dinâmico, de transformação e mudança. Quanto à noção de currículo, essa está muito vinculada a um processo estático, mero instrumento. Contudo, é preciso saber o quão dinâmico e complexo é esse conceito, podendo ser associado à ideia de 'espaço de disputa de poderes' em que os 'sujeitos são produzidos', como indicado por Santos e Paraíso (1996).

Sobre o uso das MAs na educação médica, destacamos duas considerações sobre o que foi extraído dos trabalhos lidos. A primeira é a de que, dentre as MAs mais referenciadas, estão a ABP e a Problematização. Isso significa que a análise feita pelos trabalhos, em sua maioria, diz respeito ao uso desses métodos em específico.

Uma segunda consideração a ser feita corresponde ao fato de que há uma preocupação entre os pesquisadores do tema em avaliar a mudança curricular fundamentada nas MAs e o desempenho discente com uso dessas metodologias. Isso se faz notar pelo número expressivo de trabalhos envolvidos nesse processo, quase $70 \%$ do total de produções selecionadas.

De modo geral, esses trabalhos apontam as MAs como uma possibilidade para responder aos anseios de mudança curricular dos cursos de medicina no país. As principais tentativas concretizadas atêm-se, sobretudo, a mudanças metodológicas ou pedagógicas, com a reestruturação dos currículos com base na inserção da ABP como eixo estruturante. Todavia, quando baseado apenas na mudança do método tradicional para a $\mathrm{ABP}$, esse processo apresenta lacunas e mostra não ser suficiente para mudança do perfil do egresso em medicina. Os resultados deste estudo despertam o desejo pela continuidade de investigação sobre o tema, que de modo particular e pessoal recai sobre inquietações, como: que tipos de sujeitos estariam sendo produzidos com base nos discursos que circulam em um currículo de medicina fundamentado nas MAs? Ou como esse currículo opera na produção de sujeitos? Como ele produz subjetividades? Devido à emergência e profusão de escolas médicas que 
utilizam as MAs, concluímos que mais pesquisas nesta área são necessárias para melhor compreender seu uso na educação médica.

\section{Colaboradores}

Raimundo Rodrigues de França Jr. vem realizando a pesquisa de doutorado e redigiu este artigo que dela deriva. Marlécio Maknamara é orientador da pesquisa de doutorado e contribuiu na elaboração do artigo com a organização do plano de escrita do artigo, delineamento metodológico e revisão textual.

\section{LA LITERATURA SOBRE METODOLOGÍAS ACTIVAS EN EDUCACIÓN MÉDICA EN BRASIL: NOTAS PARA UNA REFLEXIÓN CRÍTICA}

Resumen Este trabajo parte de la emergencia de escuelas médicas que fundamentan sus programas en las metodologías activas. Tuvo como objetivo realizar un mapeo en la Revista Brasileña de Educación Médica con base en la producción científica sobre conexiones entre formación médica y metodologías activas. El relevamiento bibliográfico incluyó 53 artículos, agrupados en cuatro categorías de análisis: análisis comparativa entre modelos curriculares fundamentados en el método tradicional y en las metodologías activas; evaluación del cambio curricular basado en el uso de las metodologías activas; evaluación del desempeño docente con uso de las metodologías activas; y capacitación docente en el contexto de las metodologías activas. Estos resultados señalan a las metodologías activas como propuestas que pueden dar respuestas a los anhelos de cambio curricular de las carreras de medicina en Brasil, incluso aunque aún no exista un consenso en relación a la efectividad de su uso en esta área. Sin embargo, una mirada más detallada sobre estos resultados nos permitió concluir que este proceso, cuando se basa sólo en el cambio del método de enseñanza, puede presentar lagunas y muestra no ser suficiente para cambiar el perfil del egresado en medicina. De este modo, recomendamos que se realicen más investigaciones en este campo para comprender mejor los usos de las metodologías activas en las dinámicas curriculares que fundamentan la educación médica en Brasil.

Palabras-clave educación médica; aprendizaje basado en problemas; problematización. 


\section{Notas}

1 O Programa de Incentivo às Mudanças nos Cursos de Medicina (Promed) visava oferecer apoio técnico e financeiro às escolas médicas dispostas a desenvolver processos de mudança que levassem a uma articulação com os serviços de saúde, à adoção de Metodologias Ativas de Ensino e Aprendizagem e a uma formação crítica e humanista do profissional médico (Nogueira, 2009).

2 Nas produções selecionadas para esse levantamento foi possível encontrar tanto o termo 'educação' como 'ensino' para indicar o processo de 'ensino e aprendizagem' em medicina. Todavia os autores parecem não demonstrar preocupação em referenciar um ou outro termo, fazendo o uso dos mesmos de forma abusiva. Nesse trabalho optou-se pelo uso do termo 'educação', uma vez que ao 'reduzir educação a ensino' poderíamos estar abdicando de reflexões profundas e complexas em torno de questões sociais, epistemológicas e políticas que envolvem as práticas educativas e os currículos, tal como sugerido por Oliveira et al. (2012).

3 Bordenave e Pereira no livro Estratégias de ensino-aprendizagem lançado em 1988, apresentam um esquema que define o Método do Arco de Maguerez, onde constam cinco etapas que se desenvolvem com base na realidade ou um recorte da realidade: Observação da realidade e identificação de problemas; pontos chave (em que somos levados a refletir sobre as possíveis causas da existência do problema em estudo); teorização (busca de informação sobre o problema); hipóteses de solução para os problemas encontrados e aplicação à realidade (prática).

4 Este artigo, com as devidas adaptações, contém parte do levantamento bibliográfico realizado para a tese de doutorado desenvolvida no Programa de Pós-Graduação em Educação da Faculdade de Educação da Universidade Federal da Bahia (PPGEDU/UFBA), em específico, no 'ESCRE(VI)VER: Grupo de Estudos e Pesquisas com Narrativas em Educação', coordenado pelo Prof. Dr. Marlécio Maknamara.

5 A definição desse período para realização do mapeamento não se deu de modo aleatório. O ano de 2001 foi marcado pela homologação via Conselho Nacional de Educação, das Diretrizes Curriculares do Ensino Médico, e também pelo lançamento do Programa de Incentivo às Mudanças nos Cursos de Medicina - o Promed. Ambos os acontecimentos são considerados um marco na transformação do ensino médico no Brasil. 


\section{Referências}

ALMEIDA, Enedina S.; BATISTA, Nildo A. Desempenho docente no contexto PBL: essência para aprendizagem e formação médica. Revista Brasileira de Educação Médica, v. 37, n. 2, p. 192-201. 2013.

ALMEIDA, Maria T. C.; BATISTA, Nildo A. Ser docente em métodos ativos de ensinoaprendizagem na formação do médico. Revista Brasileira de Educação Médica, v. 35, n. 4, p. 468-476. 2011

ALMEIDA, Henriqueta G. G.; FERREIRA FILHO, Olavo F. Educação permanente de docentes: análise crítica de experiências não sistematizadas. Revista Brasileira de Educação Médica, v. 32, n. 2, p. 240- 247. 2008.

ALMEIDA, Henriqueta G. G. et al. Elaborando a capacitação de tutores: subsídios da prática. Revista Brasileira de Educação Médica, v. 27, n. 3, p. 191-199. 2003.

AGUILAR-DA-SILVA, Rinaldo H. abordagens pedagógicas e tendências de mudanças nas escolas médicas. Revista Brasileira de Educação Médica, v. 33, n. 1, p. 53-62. 2009.

ANDRADE, Silvia C. et al. Avaliação do desenvolvimento de atitudes humanísticas na graduação médica. Revista Brasileira de Educação Médica, v. 35, n. 4, p. 517-525. 2011.

ANGELI, Olga A.; LOUREIRO, Sonia R. A Aprendizagem Baseada em Problemas e os recursos adaptativos de estudantes do curso médico. Revista Brasileira de Educação Médica, v. 25, n. 2, p. 32-41. 2001.

AQUILANTE, Aline G. et al. Situações-problema simuladas: uma análise do processo de construção. Revista Brasileira de Educação Médica, v. 35, n. 2, p. 147-156. 2011

ARAÚJO, Ulisses F.; SASTRE, Genoveva. Apresentação. In: ARAÚJO, Ulisses F. e SASTRE, Genoveva (orgs.). Aprendizagem baseada em problemas no ensino superior. São Paulo: Summus, 2009. p. 07-16.

ARAÚJO, Ivan de M. et al. Avaliação do aproveitamento do aluno em Nefrologia: estudo comparativo entre o método expositivo tradicional e o método de Aprendizado Baseado em Problemas. Revista Brasileira de Educação Médica, v. 25, n. 3, p. 114-125. 2001.

ARRUDA, Marina P. et al. Educação permanente: uma estratégia metodológica para os professores da Saúde. Revista Brasileira de Educação Médica, v. 32 , n. 4 , p. $518-524.2008$.

BARDIN, Laurence. Análise de conteúdo. Lisboa: Edições 70. 1977.

BARROS, Nelson F.; LOURENÇO, Lídia C. A. O ensino da Saúde Coletiva no método de Aprendizagem Baseado em Problemas: uma experiência da Faculdade de Medicina de Marília. Revista Brasileira de Educação Médica, v. 30, n. 3, p. 136-146. 2006.

BORDENAVE, Juan D.; PEREIRA, Adair M. Estratégias de ensino-aprendizagem. 28 ed. Rio de Janeiro: Vozes, 2007.

BRANDÃO, Edermeson R. M. et al. Práticas de integração Ensino-Serviço-Comunidade: reorientando a formação médica. Revista Brasileira de Educação Médica, v. 37, n. 4, p. 573-577. 2013.

BRASIL, Ministério da Educação e Cultura, Conselho Nacional de Educação (CNE). Resolução n. 3, de 20 de junho de 2014. Institui Diretrizes Curriculares Nacionais do Curso de Graduação em Medicina. Diário Oficial da República Federativa do Brasil, Brasília, 2014.

BERBEL, Neusi A. N. Metodologia da problematização: experiências com questões de ensino superior, ensino médio e clínica. Londrina: Eduel, 1998

CARABETTA JÚNIOR, Valter. Metodologia ativa na educação médica. Revista de Medicina (São Paulo). no 95, v. 3, p. 113-121, 2016. 
CEZAR, Pedro H. N. Transição paradigmática na educação médica: um olhar construtivista dirigido à Aprendizagem Baseada em Problemas. Revista Brasileira de Educação Médica, v. 34, n. 2, p. 298-303. 2010.

COSTA, Maria C. G. et al. Ensino e aprendizagem da prática profissional: perspectiva de estudantes de medicina. Revista Brasileira de Educação Médica, v. 40, n. 2, p. 245-253. 2016.

COSTA, José R. B. et al. A transformação curricular e a escolha da especialidade médica. Revista Brasileira de Educação Médica, v. 38, n. 1, p. 47-58. 2014.

COSTA, José R. B. et al. Metodologias ativas de ensino-aprendizagem: a visão de estudantes de medicina sobre a Aprendizagem Baseada em Problemas. Revista Brasileira de Educação Médica, v. 35, n. 1, p. 13-19. 2011.

CHAVES, Igor T. da S.; GROSSEMAN, Suely. O internato médico e suas perspectivas: estudo de caso com educadores e educandos. Revista Brasileira de Educação Médica, v. 31, n. 3, p. 212- 222. 2007.

CLIQUET, Marcia B.; RODRIGUES, Cibele I. S. Grupo tutorial e a saúde mental no ensino médico. Revista Brasileira de Educação Médica, v. 40, n. 4, p. 591-601. 2016.

DEWEY, John. Democracia e educação. 3. ed. São Paulo: Nacional, 1959.

DELAROSSA, Alejo. Grupos de reflexión: entrenamento institucional de coordinadores y terapeutas de grupos: Buenos Aires: Paidós; 1979.

DECKER, Isonir da R.; BOUHUIJS, Peter A. Aprendizagem Baseada em Problemas e metodologias da problematização: identificando e analisando continuidades e descontinuidades nos processo de ensino-aprendizagem. In: ARAÚJO, Ulisses F. e SASTRE, Genoveva. (orgs.). Aprendizagem baseada em problemas no ensino superior. São Paulo: Summus, 2009. p. 177-204.
FARIAS, Pablo A. M. et al. Aprendizagem ativa na Educação em Saúde: percurso histórico e aplicações. Revista Brasileira de Educação Médica, v. 39, n. 1, p. 143-158. 2015.

FARIA, Maria J. S. S. et al. Os desafios da educação permanente: a experiência do curso de Medicina da Universidade Estadual de Londrina. Revista Brasileira de Educação Médica, v. 32, n. 2, p. 248- 253. 2008.

FERREIRA, Ricardo C. et al. Aprendizagem Baseada em Problemas no Internato: há continuidade do processo de ensino e aprendizagem ativo? Revista Brasileira de Educação Médica, v. 39, n. 2, p. 276-285. 2015.

FERREIRA FILHO, Olavo F. et al. Visão docente do processo de implementação da Aprendizagem Baseada em Problemas (ABP) no Curso Médico da Universidade Estadual de Londrina (UEL). Revista Brasileira de Educação Médica, v. 26, n. 3, p. 112-120. 2002.

FREIRE, Paulo. Pedagogia do oprimido. 17. ed. São Paulo: Paz e Terra, 1987.

FREIRE, Paulo. Educação e mudança. 8. ed. São Paulo: Paz e Terra, 1999.

GERHARDT, Tatiana E. et al. Unidade 4: estrutura do projeto de pesquisa. In: GERHARDT, Tatiana Engel e SILVEIRA, Denise Tolfo (orgs.). Métodos de pesquisa. Porto Alegre: Editora da UFRGS, 2009, p. 65-89.

GIL, Antônio C. Como elaborar projetos de pesquisa. 4. ed. São Paulo: Atlas, 2002.

GOMES, Andréia P.; REGO, Sergio. Paulo Freire: contribuindo para pensar mudanças de estratégias no ensino de Medicina. Revista Brasileira de Educação Médica, v. 38, n. 3, p. 299-313. 2014.

GOMES, Andréia P.; REGO, Sergio. Transformação da Educação Médica: é possível formar um novo médico a partir de mudanças no método de ensino-aprendizagem? Revista Brasileira de Educação Médica, v 35, n. 4, p. 557-566. 2011. 
GOMES, Andréia P. Avaliação no ensino médico: o papel do portfólio nos currículos baseados em metodologias ativas. Revista Brasileira de Educação Médica, v. 34, n. 3, p. 390-396. 2010.

GOMES, Romeu et al. Aprendizagem Baseada em Problemas na formação médica e o currículo tradicional de Medicina: uma revisão bibliográfica. Revista Brasileira de Educação Médica, v. 33, n. 3, p. 444-451. 2009.

GOMES, Andréia P. et al. A Educação Médica entre mapas e âncoras: a aprendizagem significativa de David Ausubel, em busca da Arca Perdida. Revista Brasileira de Educação Médica, v. 29, n. 1, p. 105-111. 2008.

GOULART, Lúcia M. H. F. et al. Aprendizagem Baseada em Problema em microbiologia no curso de medicina da UFMG. Revista Brasileira de Educação Médica, v. 25, n. 5, p. 22-27. 2001.

KOMATSU, Ricardo S. Aprendizagem Baseada em Problemas na Faculdade de Medicina de Marília: sensibilizando o olhar para o idoso. Revista Brasileira de Educação Médica, v. 27, n. 2, p. 45-54. 2009.

LEON, Luciana B.; ONÓFRE, Fernanda de Q. Aprendizagem Baseada em Problemas na graduação médica - uma revisão da literatura atual. Revista Brasileira de Educação Médica, v. 39, n. 4, p. 614-619. 2015.

LIMA JÚNIOR, Emilton. O dilema do biscoito e o ensino universitário: uma reflexão. Revista Brasileira de Educação Médica, v. 26, n. 3, p. 211-114. 2002.

LIMA, Gerson Z.; LINHARES, Rosa E. C. Escrever bons problemas. Revista Brasileira de Educação Médica, v. 32, n. 2, p. 197-201. 2008.

LIMA, Helena M. da Costa. Experiências em buscas de informações por residentes de Medicina. Revista Brasileira de Educação Médica, v. 29, n. 1, p. 13-21. 2005.

LIMA, Gerson Z. et al. Aprendizagem Baseada em Problemas (ABP): construindo a capacitação em Londrina. Revista Brasileira de Educação Médica, v. 27, n. 1, p. 5-11. 2003.

LUNA, Willian F.; BERNARDES, Jefferson S. Tutoria como estratégia para aprendizagem significativa do estudante de Medicina. Revista Brasileira de Educação Médica, v. 40, n. 4, p. 653-662. 2015.

MAIA, José A. Metodologias problematizadoras em currículos de graduação médica. Revista Brasileira de Educação Médica, v. 38, n. 4, p. 566-574. 2014.

MAKABE, Maria L. F.; MAIA, José A. Reflexão discente sobre a futura prática médica através da integração com a Equipe de Saúde da Família na Graduação. Revista Brasileira de Educação Médica, v. 38, n. 1, p. 127-132. 2014.

MARIN, Maria J. S. et al. Aspectos das fortalezas e fragilidades no uso das Metodologias Ativas de Aprendizagem. Revista Brasileira de Educação Médica, v. 34, n. 1, p. 13-20. 2010.

MEZZARIL, Adelina. O Uso da Aprendizagem Baseada em Problemas (ABP) como reforço ao ensino presencial utilizando o ambiente de Aprendizagem Moodle. Revista Brasileira de Educação Médica, v. 35, n. 1, p. 114-121. 2011.

MINAYO, Maria C. de S. O desafio do conhecimento. 10. ed. São Paulo: HUCITEC, 2007.

MITRE, Sandra M. I. et al. Metodologias ativas de ensino-aprendizagem na formação profissional em saúde: debates atuais. Ciências e Saúde Coletiva. v. 13, n. 2, p. 2133-2144. 2008.

MORÉ, Nilson C.; GORDAN, Pedro A. A percepção dos professores do Departamento de Medicina da Universidade Estadual de Maringá sobre suas dificuldades e necessidades educacionais para o desenvolvimento do ensino médico. Revista Brasileira de Educação Médica, v. 28, n. 3, p. 91-100. 2004.

MORAES, Magali A. A.; MANZINI, Eduardo J. Concepções sobre a Aprendizagem Baseada em Problemas: um estudo de caso na Famema. 
Revista Brasileira de Educação Médica, v. 30, n. 3, p. 125- 135. 2006.

NOVAES, Maria R. C. G. et al. Actitudes éticas de los estudiantes y egresados en carrera de medicina con metodologias activas. Revista Brasileira de Educação Médica, v. 34, n. 1, p. 43-56. 2010.

NOGUEIRA, Maria I. As mudanças na educação médica brasileira em perspectiva: reflexões sobre a emergência de um novo estilo de pensamento. Revista brasileira de educação médica, v. 33, n. 2, p. 262-270. 2009.

NUNES, Cassia R. R.; NUNES, Amauri P. Aportes teóricos da Ação Comunicativa de Habermas para as Metodologias Ativas de Aprendizagem. Revista Brasileira de Educação Médica, v. 29, n. 2, p. 179-184. 2005.

OLIVEIRA, Inês B. et al. Relações entre educação e ensino a partir do campo do currículo. In: Anais da $35^{a}$ Reunião anual da Associação Nacional de Pós-graduação e Pesquisa em Educação; 2012; Porto de Galinhas-PE; ANPED, 2012. Disponível em: https://www.fe.unicamp.br/ gtcurriculoanped/35RA/trabalhos/TE-Anped2012Rel_educacao_ensino.pdf. Acesso em: 21 out. 2017.

OLIVEIRA, Vanessa T. D.; BATISTA, Nildo A. Avaliação Formativa em Sessão Tutorial: Concepções e Dificuldades. Revista Brasileira de Educação Médica, v. 36, n. 3, p. 374-380. 2012.

PEIXOTO, José M. et al. Atitude do estudante de Medicina a respeito da relação médico-paciente $\mathrm{x}$ modelo pedagógico. Revista Brasileira de Educação Médica, v. 35, n. 2, p. 229-236. 2011.

PENAFORTE, Julio C. John Dewey e as raízes filosóficas da aprendizagem baseada em problemas. In: MAMEDE, Sílvia; PENAFORTE, Julio C. (orgs.). Aprendizagem baseada em problemas: anatomia de uma nova abordagem educacional. São Paulo: Hucitec/ ESP-CE, 2001. p. 49-78.

PUTNAM, A. R. Problem-based teaching and learning in educational technology. Paper apresentado na 75 Conferencia Annual da Asso- ciaciation for Carrer and Technical Education, New Orleans, 13-16 dez. 2001. Disponível em: https://files.eric.ed.gov/fulltext/ED465039.pdf. Acesso em: 10 jul. 2018.

REGO, Cinthia; BATISTA, Sylvia H. Desenvolvimento Docente nos Cursos de Medicina: um Campo Fecundo. Revista Brasileira de Educação Médica, v. 36, n. 3, p. 317-324. 2012.

SANTOS, Lucíola L. P.; PARAÍSO, Marlucy A. Dicionário crítico da educação: Currículo. Presença Pedagógica, v. 2, n. 7, p. 82-84. 1996.

SENA, Tais S.; COSTA, Mariana L. Reflexões sobre a inserção da temática Gestão da Clínica na formação profissional em Saúde. Revista Brasileira de Educação Médica, v. 40, n. 2, p. 278-285. 2016.

SILVA, Sonia L. et al. Estratégia educacional baseada em problemas para grandes grupos: relato de experiência. Revista Brasileira de Educação Médica, v. 39, n. 4, p. 607-613. 2015.

SMOLKA, Maria L. R. M. et al. Autonomia no contexto pedagógico: percepção de estudantes de Medicina acerca da Aprendizagem Baseada em Problemas. Revista Brasileira de Educação Médica, v. 38, n. 1, p. 5-14. 2014.

SOUZA, Fábio G. de M.; MENEZES, Maria da G.C. Estresse nos estudantes de Medicina da Universidade Federal do Ceará. Revista Brasileira de Educação Médica, v. 29, n. 2, p. 91-96. 2005.

TAROCO, Ana P. R. M. et al. Currículo orientado por competência para a compreensão da integralidade. Revista Brasileira de Educação Médica, v. 41, n. 1, p. 12-21. 2017.

TENÓRIO, Leila P. et al. Saúde mental de estudantes de escolas médicas com diferentes modelos de ensino. Revista Brasileira de Educação Médica, v. 40, n. 4, p. 574-582. 2016.

TURINI, Barbosa; ALMEIDA, Marcio J. Os professores de Medicina e o ensino de graduação 
extramuros. Revista Brasileira de Educação Médica, v. 26, n. 3, p. 151-161. 2002.

VARGAS, Lúcia H. M. et al. Inserção das ciências básicas no currículo integrado do curso de Medicina da Universidade Estadual de Londrina. Revista Brasileira de Educação Médica, v. 32, n. 2, p. 174-179. 2008.

VENTURELLI, José; FIORINI, Vania M. L. Programas educacionais inovadores em escolas médicas: capacitação docente. Revista Brasileira de Educação Médica, v. 24, n. 3, p. 7-21. 2001.
VILA, Ana C. D.; VILA, Vanessa S. C. Tendências da produção do conhecimento na educação em saúde no Brasil. Revista Latino-americana de Enfermagem, v. 15, n. 6, p. 177-190, 2007.

WIERZCHON, Patricia M. “O ensino médico no Brasil está mudando?" Transpondo desafios para concretizar mudanças. Revista Brasileira de Educação Médica, v. 26, n. 1, p. 62-66. 2002. 